\title{
Age Related Oxidative Changes in Primary Porcine Fibroblasts Expressing Mutated Huntingtin
}

Petra Smatlikova ${ }^{1,3}$, Georgina Askeland ${ }^{4,5}$, Michaela Vaskovicova ${ }^{2,3}$, Jiri Klima ${ }^{1}$, Jan Motlik ${ }^{1}$, Lars Eide $^{4}$, Zdenka Ellederova ${ }^{{ }^{*}}$

${ }^{1}$ Laboratory of Cell Regeneration and Plasticity, Research Center PIGMOD, Institute of Animal Physiology and Genetics, Czech Academy of Science, Libechov, Czech Republic

${ }^{2}$ Laboratory of DNA Integrity, Research Center PIGMOD, Institute of Animal Physiology and Genetics, Czech Academy of Science, Libechov, Czech Republic

${ }^{3}$ Department of Cell Biology, Faculty of Science, Charles University in Prague, Prague, Czech Republic

${ }^{4}$ Department of Medical Biochemistry, University of Oslo and Oslo University Hospital, Oslo, Norway

${ }^{5}$ Department of Microbiology, Oslo University Hospital, Oslo, Norway

*correspondence author:

Zdenka Ellederova,

Address: Rumburska 89, 27721 Libechov, Czech Republic

Tel: +420315639565

email: ellederova@iapg.cas.cz

Petra Smatlikova

Georgina Askeland

Michaela Vaskovicova

Jiri Klima

Jan Motlik

Lars Eide email: smatlikova@iapg.cas.cz

email: georgina.ruth.adeline.askeland@rr-research.no

email: vaskovicova@iapg.cas.cz

email: klima@iapg.cas.cz

email: motlik@iapg.cas.cz

email: lars.eide@medisin.uio.no 


\begin{abstract}
Background: Huntington's disease (HD) is a devastating neurodegenerative disorder caused by CAG triplet expansions in the huntingtin gene. Oxidative stress is linked to HD pathology, although it is not clear whether this is an effect or mediator of disease. The transgenic minipig (TgHD) expresses the $\mathrm{N}$-terminal part of human mutated huntingtin and represents a unique model to investigate therapeutic strategies towards HD, and more detailed characterization of this model is needed to fully utilize its potential.
\end{abstract}

Methods: In this study, we focused on molecular and cellular features of fibroblasts isolated from TgHD minipigs and the wild type (WT) siblings at different ages, pre-symptomatic $24-36$ months old and with behavioural symptoms onset at the age of 48 months. We measured oxidative stress, the expression of oxidative stress related genes, proliferation capacity along with the expression of cyclin B1 and D1 proteins, cellular permeability, and nuclear and mitochondrial DNA integrity in these cells.

Results: TgHD fibroblasts isolated from 48 months old animals showed increased oxidative stress, which correlate with overexpression of SOD2 encoding mitochondrial superoxide dismutase 2, and NEIL3 gene, encoding DNA glycosylase involved in replication associated repair of oxidized DNA. TgHD cells displayed abnormal proliferation capacity and permeability. We further demonstrated an increased level of nuclear DNA damage in pre-symptomatic TgHD fibroblasts (isolated from 24 -36 months old animals).

Conclusions: Our results unravel phenotypic alterations in primary fibroblasts isolated from $\mathrm{TgHD}$ minipig model at the age of 48 months. Importantly, nuclear DNA damage appears to precede these phenotypic alterations. Our results highlight the impact of fibroblasts from TgHD minipigs in studying molecular mechanisms occurring gradually in HD pathophysiology with age.

\title{
Keywords
}

Huntington's disease - oxidative stress - proliferation - permeability - minipig model - large animal model - mutated huntingtin - primary fibroblasts - DNA damage 


\section{Background}

Huntington's disease (HD) is an inherited neurodegenerative disorder caused by the CAG expansion (CAG > 36) in a gene encoding huntingtin (HTT) protein, which has a role in many cellular processes. The pathogenesis of HD is not well understood. Molecular events, such as protein aggregation, mitochondrial dysfunction, and transcriptional dysregulation have been linked to HD pathogenesis [1].

During the aggregation process, huntingtin starts to form an aggregation core followed by forming fibrillar structures and later insoluble aggregates or inclusions that increase with age [2]. It has been shown that aggregation nuclei are composed mostly by the $\mathrm{N}$-terminal fragments of mutated huntingtin, and that the presence of $\mathrm{N}$-terminal fragments increases the formation of aggregates [35].

To facilitate studies of pathogenesis and therapy of HD, an unique transgenic minipig model was generated by microinjection of a lentiviral vector encoding the N-terminal (548 aa) part of human HTT containing $126 \mathrm{CAG} / \mathrm{CAA}$ repeats under the control of the human HTT promoter [6]. The slow progression of the disease in this model combined with the closer phylogenetic relation to humans as compared to rodent HD models, makes it highly interesting for studying HD progression and therapeutic interventions [6-11]. Recently, the age of onset for motor, behaviour and cognitive performance was determined to be 48 months [12].

Expression of mutant huntingtin (mtHTT) leads to selective cellular dysfunction and degeneration [13]. Oxidative stress has been considered as one of the key players in HD disease progression $[14,15]$. Though, it is still not clear whether oxidative stress is a cause or effect in HD [16]. Reactive oxygen species (ROS), are well established as signalling molecules involved in a variety of signal transduction pathways such as normal proliferation and differentiation, apoptotic pathway, cell survival pathway, growth factor signalling, transcriptional processes, and others [16-18].

Mitochondrial dysfunction is another feature associated with HD pathogenesis [19-22]. Previous studies have shown disturbances of mitochondrial metabolism in the HD brain, skeletal muscle, or even cultivated skin fibroblasts [23]. Moreover, it has been suggested that mitochondrial dysfunction in HD is directly caused by oxidative stress induced by mtHTT [24]. Peripheral blood mononuclear cells (PBMCs) from HD patients displayed reduced levels of key mitochondrial parameters, yet, the electron transport chain biochemical complex activities were unaffected [25]. In the TgHD minipig model we observed genotype-specific effects on mitochondrial DNA (mtDNA) damage, mtDNA copy number, 8-oxoguanine DNA glycosylase activity, and global level of the epigenetic marker 5-methylcytosine in basal ganglia [12].

Links between DNA repair defects and neurodegenerative diseases have been known for many years. Fibroblasts and lymphocytes cultured from patients with HD, Alzheimer's disease, Parkinson's disease and Amyotrophic lateral sclerosis have all been shown to be sensitive to DNA damage induced by ionising radiation or exogenous chemical mutagens [26-28]. It has been suggested that accumulation of DNA damage as a result of inadequate DNA repair could cause neurodegeneration, although it has been difficult to discriminate between this hypothesis and the accrual of DNA damage caused by other pathological cellular dysfunctions [27].

Here we investigated oxidative stress, DNA damage, and other cellular and molecular events in primary fibroblasts isolated from minipigs transgenic for the N-terminal part of human mutated huntingtin (TgHD) and their wild type (WT) controls at pre-symptomatic stage and at the age of behavioural symptoms onset. 


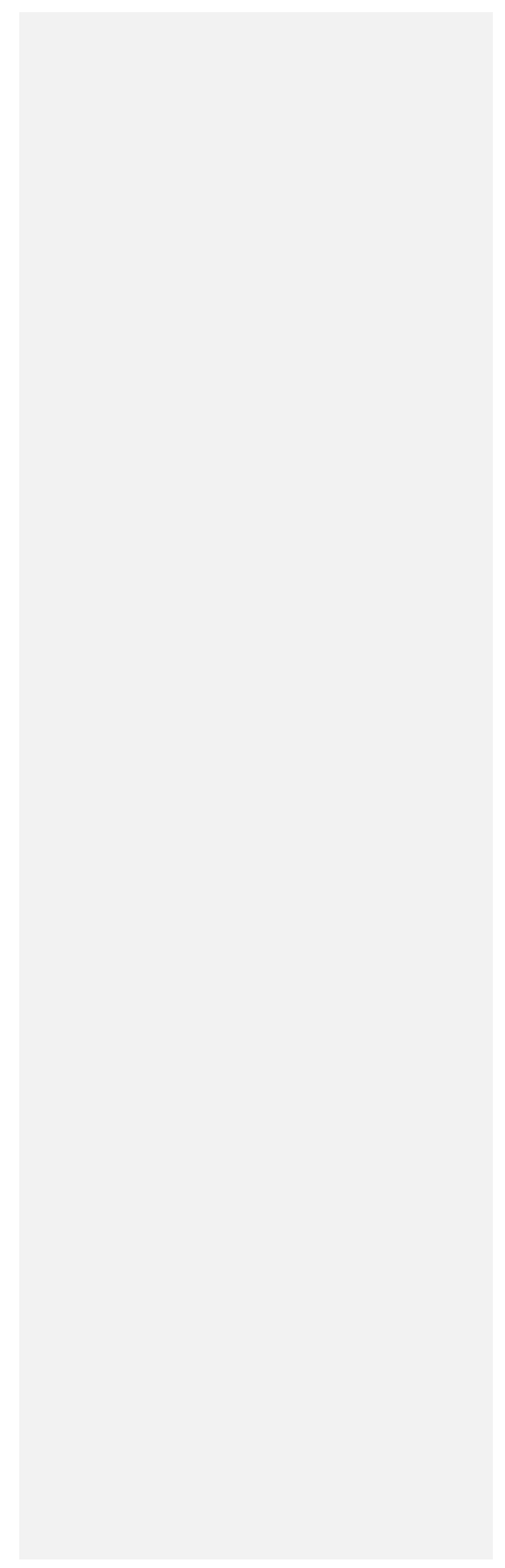




\section{Methods}

\section{Experimental animals}

Wild type (WT) and transgenic (TgHD) minipigs for HD were bred according to the Animal Care and Use Committee of Institute of Animal Physiology and Genetics and current Czech regulations and guidelines for animal welfare with approval by the State Veterinary Administration of the Czech Republic. In this study, fibroblasts from pre-symptomatic age (7 WT + 7 TgHD; age 24-36 months) and symptomatic age ( 4 WT +4 TgHD; age 48 months) both males and females (similar numbers of males and females in the groups) were used. The power analysis of the sample size based on the prediction of at least $3,2 \%$ difference in mean showed 4 animals in each group to be sufficient for the power $90 \%$ and the alpha level 0,01 .

\section{Primary fibroblast preparation and cultivation}

Primary fibroblasts were isolated from pig skin grafts. In the second passage, cells were cryopreserved in MEM alpha medium (Gibco, \#22561) with 10\% DMSO (Sigma-Aldrich, \#D8418), 25\% FBS and stored in liquid nitrogen until used. After thawing the cells in $37^{\circ} \mathrm{C}$ water bath for maximum 2 minutes, cells were routinely cultured as monolayers with MEM alpha without nucleotides (Gibco, \#22561) supplemented with 10\% FBS (Sigma-Aldrich, \#F7524) and antibiotics $(0,1 \%$ Gentamicin, Sigma-Aldrich, $\# \mathrm{G} 1397)$ at $37^{\circ} \mathrm{C}$ in a humidified incubator with $5 \% \mathrm{CO}_{2}$.

In the fifth passage cells were plated in $75 \mathrm{~cm}^{2}$ TPP flasks at the number of $1 \times 10^{6}$ cells per flask Cells were then cultured in MEM alpha (10\% FBS and antibiotics) under normal culture conditions $\left(37^{\circ} \mathrm{C}, 5 \% \mathrm{CO}_{2}\right.$ in humidified incubator) for 3 days. In the third day, monolayer of cells was washed with PBS and detached by $0,4 \%$ trypsin in PBS for 4 minutes. Cells were then collected in $15 \mathrm{~mL}$ tubes with media and centrifuged at $1200 \mathrm{~g}$ for 3 minutes. Afterwards, the media was aspirated, and the cell pellets were resuspended in $10 \mathrm{~mL}$ of fresh media. Cells were then counted using automatic Vi-CELL $^{\mathrm{TM}}$ (Backman Coulter Life Sciences) cell viability analyser. Only viable cells were included into the analysis. All direct counting experiments were done in four replicates. All experiments were performed in the third day of the fifth passage.

\section{Reactive oxygen species (ROS), lipid peroxidation and permeability measurements}

ROS were measured by CellROX Deep Red Reagent (Thermo Fisher Scientific, \#C10422), lipid peroxidation was measured by Image-iT (Thermo Fisher Scientific, \#C10445) and permeability was measured by Calcein AM (Thermo Fisher Scientific, \#C1430).

Cells were plated in 24-well plates (25000 cells per well) and cultured in MEM alpha (10\% FBS and antibiotics) under normal culture conditions $\left(37^{\circ} \mathrm{C}, 5 \% \mathrm{CO}_{2}\right.$ in humidified incubator). In the third day of culture cells were incubated with $5 \mu \mathrm{M}$ CellROX Deep Red Reagent or $10 \mu \mathrm{M}$ ImageiT or $1 \mu \mathrm{M}$ Calcein $\mathrm{AM}$ for 30 minutes at $37^{\circ} \mathrm{C}$. Cells were then washed once with fresh media, incubated for 2 hours at $37^{\circ} \mathrm{C}$ (CellROX and Calcein AM) or 30 minutes (Image-iT) and fluorescence was measured by fluorescent spectrophotometer at specific wavelengths. Cells were then incubated with Hoechst (Thermo Fisher Scientific, \#H3570) at the concentration of 1:2000 in fresh media for 10 minutes, washed once with PBS and read by fluorescent spectrophotometer.

Fluorescent excitation wavelength of $640 / 10 \mathrm{~nm}$ for CellROX, 488/10 nm for oxidized Image-iT, $577 / 10 \mathrm{~nm}$ for reduced Image-iT, 495/10 nm for Calcein AM, and 350/20 nm for Hoechst was used and fluorescent emission was measured at $665 / 10 \mathrm{~nm}$ for CellROX, $510 / 10 \mathrm{~nm}$ for oxidized ImageiT, 595/10 nm for reduced Image-iT, 515/10 nm for Calcein AM, and 460/20 nm for Hoechst. 


\section{SOD2 activity assay}

Cells were lysed in ice cold $0,1 \mathrm{M}$ TRIS/HCL $\mathrm{pH} 7,4$ containing $0,5 \%$ Triton X-100, $5 \mathrm{mM} \beta$-ME, $0,1 \mathrm{mg} / \mathrm{mL}$ PMSF, sonicated for $15 \mathrm{mins}$ and centrifuged at $600 \mathrm{~g}$ for $10 \mathrm{mins}$ at $4^{\circ} \mathrm{C}$. To obtain only mitochondrial fraction in order to enrich SOD2 concentration over the other SOD isoforms supernatants were centrifuged at $15000 \mathrm{~g}$ for $5 \mathrm{mins}$ at $4^{\circ} \mathrm{C}$. The sediments with mitochondrial fractionwere resuspended in lysis buffer and the protein concentrations were measured using Pierce $660 \mathrm{~nm}$ protein assay kit (Thermo Scientific, \#22660). Equal protein concentration $(1 \mu \mathrm{g} / \mathrm{mL})$ of samples were loaded to 96-well plate in duplicates and assayed using SOD determination kit (Sigma-Aldrich, \#19160) according to manufacturer's protocol. The absorbance was measured at $450 \mathrm{~nm}$ by spectrophotometer and the SOD activity was calculated using the equation provided by manufacturer.

\section{Gene expression analyses}

Total cellular RNA was isolated from cell pellets using RNeasy Plus Mini Kit (Qiagen, \#74134) and reverse transcribed using High-Capacity cDNA RT Kit (Thermo Fisher Scientific, \#4368814). After cDNAs were PCR amplified, SYBR Green (Thermo Scientific, \#A25780) and specific primers designed for Sus Scrofa (table 1) were used in quantitative real-time PCR according to the manufacturer's protocol using CFX96 Touch Real-Time PCR Detection System (Bio-Rad). Relative quantification of gene expression was calculated following equation: $2^{\wedge}-\Delta \Delta \mathrm{Ct}$.

Table 1. Primers used for gene expression analyses

\begin{tabular}{|l|l|l|}
\hline Target & Forward & Reverse \\
\hline$A C T B$ & 5'-GAGAAGCTCTGCTACGTCG-3' & 5'-CCAGACAGCACCGTGTTGG-3' \\
\hline$B 2 M$ & 5'-AAACGGAAAGCCAAATTACCTGA-3' & 5'-ATCTCTGTGATGCCGGTTAGTG-3' \\
\hline$P G C 1 A$ & 5'-TCAGTCCATTGGTGAGGACAG-3' & 5'-CTGGAGACAGGGTGTCTGTAATA-3' \\
\hline$N E I L 2$ & 5'-CGCCCAGGTCCATGGAAAG-3' & 5'-CTCTGACAGTGGGCTGCTAC-3' \\
\hline SOD2 & 5'-GCAAGGAACAACAGGTCTGG-3' & 5'-CGGCGTATCGCTCAGTTACA-3' \\
\hline LDHA & 5'-CCTAATGGGGGAAAGGCTGG-3' & 5'-CGCTCCATACAGGCACACT-3' \\
\hline$P G K 1$ & 5'-ATGCTTCTGGGAGCAAGGTTA-3' & 5'-CGGTGAGCAGTACCAAAAGC-3' \\
\hline$V E G F$ & 5'-GTGCCCACTGAGGAGTTCAA-3' & 5'-GGCCCACAGGGATTTTCTTG-3' \\
\hline$U C P 2$ & 5'-TACAAAGGGTTCACGCCCTC-3' & 5'-GCCCTCTTCAGCTGCTCATA-3' \\
\hline$C Y T B$ & 5'-GTAGGCCTCAATGTTCCTGCT-3' & 5'-TCGACATGGCCAACACATCA-3' \\
\hline$N D 1$ & 5'-AGCCACATCCTCAATCTCC-3' & 5'-CCCGATGAGTGCGTATTTT-3' \\
\hline$P D K 1$ & 5'-TGTGAAGATGAGTGACCGAGG-3' & 5'-AGGCGTGATATGGGCAATCC-3' \\
\hline$N E I L 3$ & 5'-GGGCCAAAAGCTTTACGGATT-3' & 5'-CAGAGCATTCGTCCTCCCTG-3' \\
\hline$H K 2$ & 5'-GCTCAACCATGACCAAGTGC-3' & 5'-AACTCTCCGTGTTCTGTCCC-3' \\
\hline
\end{tabular}

\section{Nuclear and mitochondrial DNA damage}

DNA was isolated from cell pellets using DNeasy Blood and Tissue Kit (Qiagen, \#69506) following the manufacturers protocol with minor modifications. Quantification of DNA was carried out by spectrophotometric analysis (BioTek, EPOCH ${ }^{\mathrm{TM}}$ ) and adjusted to desired concentration for downstream analysis. DNA integrity analysis was carried out using the RADF (Real-time qPCR Analysis of Damage Frequency) method as previously described [29]. Briefly, 6ng and 30ng of DNA (for mtDNA and nDNA respectively) was added to non-Taq $\alpha$ and Taq $\alpha$ containing reaction mixtures. Damage frequency was calculated based on the differences between non-Taq $\mathrm{I}$ 
and Taq $\alpha$ reactions using the following equation: $2^{\wedge}-\left(\mathrm{Ct}^{\mathrm{Taq}}-\mathrm{Ct}^{\mathrm{nt}}\right)$ Primers designed for MT-RNR1 and NDUFA9 were used to represent mtDNA and nDNA (table 2).

Table 2. Primers used for nDNA and mtDNA

\begin{tabular}{|c|c|c|}
\hline Target & Forward & Reverse \\
\hline $\begin{array}{l}\text { nDNA damage } \\
(N D U F A 9)\end{array}$ & $\begin{array}{l}\text { 5'- } \\
\text { GTTGTGAATGGTGCTAACTGCT- } \\
\text { 3' }\end{array}$ & $\begin{array}{l}5^{\prime}- \\
\text { ACCAGAGACAATAAAGCAGAGGAG- } \\
3^{\prime}\end{array}$ \\
\hline $\begin{array}{l}\text { mtDNA damage } \\
(M T-R N R 1)\end{array}$ & 5'-TCGCAACTGCCTAAAACTCA-3' & 5'-GAATTGGCAAGGGTTGGTAA-3' \\
\hline
\end{tabular}

\section{Western blot analyses}

Total extracts were prepared by lysing cells in RIPA buffer $(150 \mathrm{mM} \mathrm{NaCl}, 5 \mathrm{mM}$ EDTA pH 8 , $0,05 \%$ NP- $40,1 \%$ sodium deoxycholate, $0,1 \%$ SDS, $1 \%$ Triton X-100, $50 \mathrm{mM}$ Tris- $\mathrm{HCl} \mathrm{pH} 7,4)$ supplemented with protease and phosphatase inhibitors (Thermo Fisher Scientific, \#78445) sonicated for $15 \mathrm{~min}$, and centrifuged at $20000 \mathrm{~g}, 15 \mathrm{~min}$, at $4^{\circ} \mathrm{C}$. Protein concentrations were measured directly using Take3 (Synergy HTX, BioTek). Immunoblottings were performed with cell extracts of total protein concertation $15-20 \mu \mathrm{g}$, boiled in loading buffer, loaded onto $4-12 \%$ NuPAGE Bis-Tris gel (Invitrogen, Thermo Fisher Scientific, \#NP0335BOX, \#NW04122BOX) or 3 - 8\% Tris-Acetate gel (Invitrogen, Thermo Fisher Scientific, \#EA03752BOX, \#EA03755BOX) and separated with SDS-PAGE (150 V for 1,5 hour). Proteins were transferred using iBlot Gel Transfer Device to nitrocellulose membrane. After blocking in $1 \%$ or $5 \%$ skimmed milk or $5 \%$ BSA, membranes were incubated with primary antibody overnight at $4{ }^{\circ} \mathrm{C}$ and with secondary antibody for 1 hour at RT. Anti-cyclin B1 (\#MS-338-P1, 1:500) was purchased from Thermo Fisher Scientific, anti-cyclin D1 (\#92G2, 1:1000) from Cell Signaling and anti-HTT (\#EPR5526, 1:3000) from Abcam. Secondary antibody conjugated with HRP anti-mouse (Jackson ImmunoResearch, \#711-035-152, 1:10000) or anti-rabbit (Jackson ImmunoResearch, \#711-035-152, 1:10000) was used. The signal was detected using an ECL kit (GE Healthcare Life Sciences, \#RPN2232) and ChemiDoc XRS+ system (Biorad). Loading of samples was normalized to the total lane protein obtained by Pierce ${ }^{\mathrm{TM}}$ Reversible Protein Stain Kit for Nitrocellulose Membranes (Thermo Scientific, \#24580) performed directly after protein transfer and prior blocking the membrane. Results were quantitated using Image Lab ${ }^{\mathrm{TM}}$ Software (Biorad).

\section{Cellular viability test}

Cells were plated in 24-well plates (25000 cells per well) and cultured in MEM alpha (10\% FBS and antibiotics) under normal culture conditions $\left(37^{\circ} \mathrm{C}, 5 \% \mathrm{CO}_{2}\right.$ in humidified incubator). In the third day of culture medium was replaced by Alamar Blue reagent (Thermo Fisher Scientific, \#DAL1025) resuspended in culture medium at the concentration of 1:10 and cultured for 4 hours under normal culture condition. Fluorescence was read by fluorescent spectrophotometer. Cells were then washed once with PBS and incubated with Hoechst (Thermo Fisher Scientific, \#H3570) in PBS at the concentration of 1:2000 for 10 minutes and read by fluorescent spectrophotometer. Fluorescent excitation wavelength of $560 / 20 \mathrm{~nm}$ for Alamar Blue, and $350 / 20 \mathrm{~nm}$ for Hoechst was used and fluorescent emission was read at 595/20 nm for Alamar Blue, and 460/20 nm for Hoechst.

\section{Statistical analysis}


Data were statistically analysed using GraphPad Prism software. Statistical significance was determined by Student's $t$ test or two-way ANOVA followed by post hoc Sidak comparison test

Statistical test used in each case is indicated in the figure legend. All graphs indicate mean with SEM. 


\section{Results}

\section{Elevated oxidative stress in primary porcine fibroblasts expressing mtHTT}

In order to characterize noninvasively the progression of HD in cells of the large animal model, the minipig encoding the N-terminal part of human mutated huntingtin (124Q), we isolated primary fibroblasts from these TgHD minipigs and their WT controls at pre-symptomatic age (24 - 36 months old) (hereby termed pre-symptomatic) and at the age of 48 months (symptomatic). The expression of huntingtin protein in these cells was determined by Western blot analysis (Fig. 1A). The mtHTT represents the N-terminal fragment of human mutated protein containing the polyQ tract, with an estimated size of $110 \mathrm{kDa}$. In comparison, minipig endogenous HTT (enHTT) has a molecular weight of approximately $348 \mathrm{kDa}$, (Fig. 1A). The expression of mtHTT was stable during aging and across generations.

There is a strong evidence that oxidative stress is occurring in HD progression [30-33]. In order to investigate the role of oxidative stress in $\mathrm{HD}$, WT and TgHD fibroblasts representing presymptomatic and symptomatic age were analysed for intracellular reactive oxygen species (ROS) level. Cells were incubated with Cell ROX, the ROS-sensitive fluorescent probe, and recorded at 120 minutes time point to determine the ROS generation. There was no significant difference in ROS generation between WT and TgHD at the pre-symptomatic age. However, TgHD fibroblasts from 48 months minipigs displayed higher ROS levels than age-matched WT fibroblasts. $(P=0.003, t$ test $)$. In fibroblasts obtained from younger animals the level of ROS was only slightly higher in TgHD cells compared to WT without any significance (Fig. 1B). Next, we performed ratiometric lipid peroxidation test to determine the oxidative stress in these cells. There were significantly higher levels of oxidative stress in TgHD cells isolated from pre-symptomatic animals (two-way ANOVA, Genotype $P=0.0007, F=17.47$; Sidak post hoc test $P=0.004$ ), as well as in TgHD cells from 48 months old animals (Sidak post hoc test $P=0.027$ ) compared to age-matched WT cells (Fig. 1C). These data indicate elevated oxidative stress in primary TgHD fibroblasts expressing mutated huntingtin.

The difference in intracellular redox levels motivated investigation of key genes associated with oxidative stress, metabolism and DNA repair (Supplementary Fig. S1). Two genes were found to be specifically upregulated in TgHD fibroblasts of symptomatic age: the DNA repair gene nei like DNA glycosylase 3 (NEIL3), and the mitochondrially localized manganese superoxide dismutase (SOD2). NEIL3 was upregulated approximately $40 \%$ in TgHD fibroblasts at the age of 48 months compared to WT cells isolated from age-matched controls ( $P=0.045, t$ test) (Fig. 1D) while there was no difference at younger age (Fig. 1D). As NEIL3 is involved in repair of oxidized DNA, the upregulation might be an indication of an underlying oxidative stress (Fig. 1B-C). Along with this correlation, there was also a significant upregulation of SOD2 expression in TgHD fibroblasts from 48-months-old minipigs compared to those from WT at similar age ( $P=0.003, t$ test) (Fig. 1E). Like the situation for NEIL3 expression and ROS levels, there was no difference between TgHD and WT fibroblasts with respect to expression of SOD2 when isolated from younger animals compared to their age-matched WT controls (Fig. 1E). 

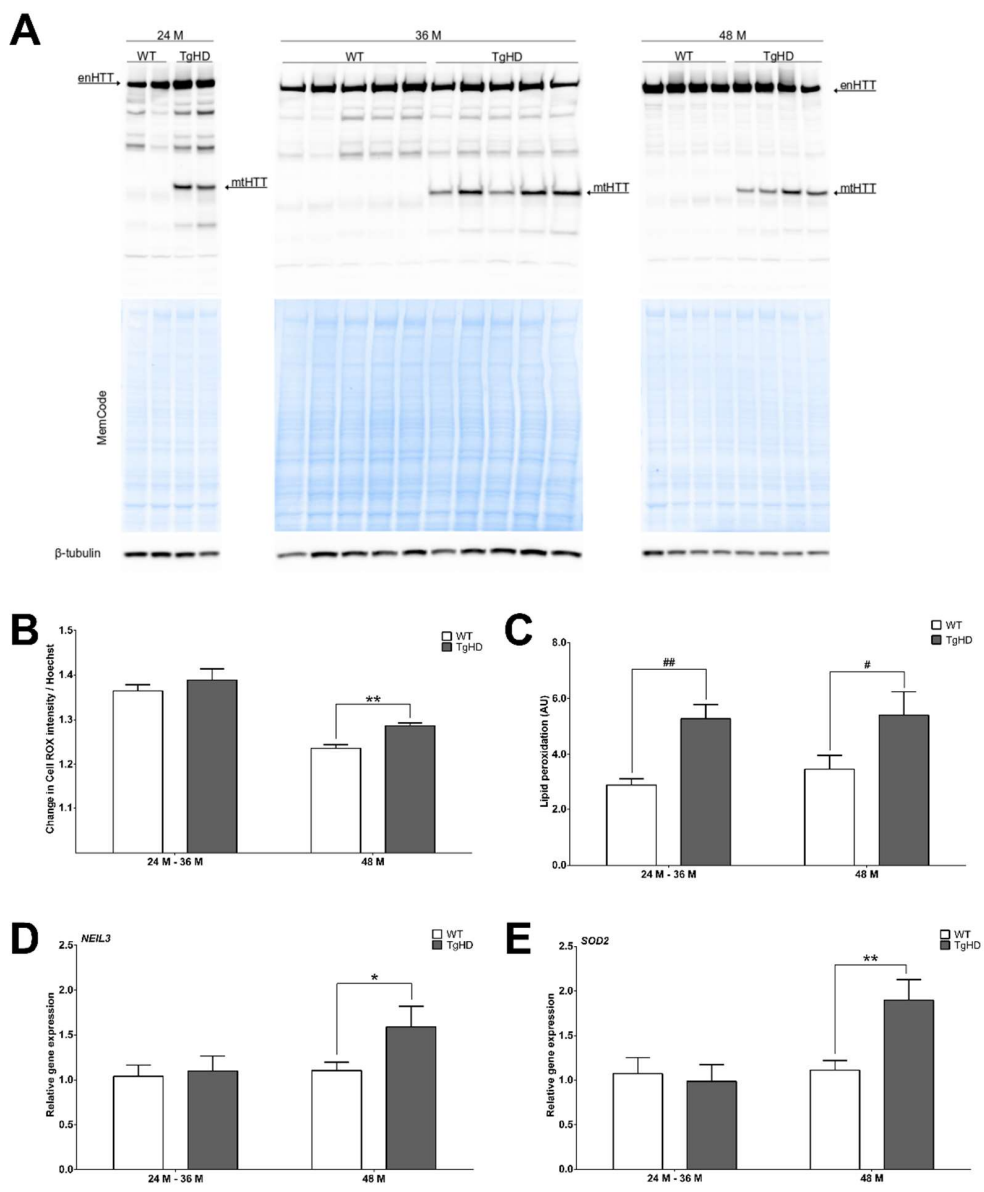

Fig. 1 Increased oxidative stress in fibroblasts isolated from TgHD minipigs at the age of 48 months

(A) Western blot analysis with anti-huntingtin antibody of primary porcine fibroblasts isolated from wild type (WT) and transgenic (TgHD) animals at the pre-onset age ( $24 \mathrm{M}, \mathrm{n}=2 \mathrm{WT}$ and $2 \mathrm{TG} ; 36 \mathrm{M}, \mathrm{n}=5$ WT and 5 TgHD) and at the age of 48 months ( $48 \mathrm{M}, \mathrm{n}=4 \mathrm{WT}$ and $4 \mathrm{TgHD})$ to show the expression of endogenous huntingtin (enHTT, $348 \mathrm{kDa}$ ) and fragment of mutated huntingtin (mtHTT, $110 \mathrm{kDa})$. (B) Measurement of ROS in fibroblasts from WT and TgHD animals at the pre-onset age (24 M - 36 M, n=7 WT and 7 TgHD) and at the age of 48 months ( $48 \mathrm{M}, \mathrm{n}=4 \mathrm{WT}$ and $4 \mathrm{TgHD}$ ). Values in graph indicate mean of the change in CellROX intensity normalised to Hoechst intensity with SEM. ${ }^{* *} P<0.01, t$ test. (C) Measurement of lipid peroxidation in fibroblasts isolated from WT and TgHD animals at the pre-onset age $(24 \mathrm{M}-36 \mathrm{M}, \mathrm{n}=6 \mathrm{WT}$ and $6 \mathrm{TgHD}$ ) and at the age of 48 months ( $48 \mathrm{M}, \mathrm{n}=4 \mathrm{WT}$ and $4 \mathrm{TgHD})$. Values in graph indicate mean of the ration of reduced and oxidized Image-iT fluorescence intensity normalised to Hoechst intensity with SEM. $\# P<0.05, \# \#<0.01$ two-way ANOVA followed by post hoc Sidak comparison test. (D) Relative gene expression of NEIL3 and (E) SOD2 in primary porcine fibroblasts isolated from WT and TgHD animals at the pre-onset age ( $24 \mathrm{M}-36 \mathrm{M}, \mathrm{n}=7 \mathrm{WT}$ and $7 \mathrm{TgHD}$ ) and at the age of 48 months ( $48 \mathrm{M}, \mathrm{n}=4 \mathrm{WT}$ and 4 TgHD). Values in graph indicate mean of relative gene expression with SEM. $* P<0.05$, $* * P<0.01, t$ test. 


\section{Altered proliferation in primary $\mathrm{TgHD}$ fibroblasts at the age of 48 months}

During regular passaging and culturing the cells we noticed differences in proliferation capacity between TgHD and WT fibroblasts. Direct counting revealed significantly higher number of viable cells from 48-month-old TgHD animals compared to age matched WT control cells (two-way ANOVA, Genotype $P=0.021, F=5.610$; Sidak post hoc test $P=0.038$ ) (Fig. 2A). No difference was observed in fibroblasts isolated from younger animals (Fig. 2A). We assume that primary porcine fibroblasts isolated from WT animals display normal age-related reduced proliferation capacity (two-way ANOVA, Age $P=0.007, F=7.843$; Sidak post hoc test WT $-P=0.003$ ), whereas those from TgHD minipigs display abnormal proliferation.

Following these results, we examined the expression of cell cycle regulatory proteins, cyclin B1 and cyclin D1 by Western blot analysis. Western blot analysis revealed markedly decreased expression of cyclin B1 in the asynchronous population of TgHD cells isolated at the age of 48 months old animals compared to their WT controls ( $P=0.039, t$ test $)$ (Fig. 2B). In the population of cells isolated from younger animals we did not observe any significant differences in cyclin B1 and cyclin D1 expression. We detected only slightly (non-significant) decreased levels of cyclin D1 in the population of cells obtained from 48 months old WT animals (Fig. 2B). Since cyclin B1 regulates cell cycle and activates "mitochondrial checkpoint" initiating cell cycle delay [34] decreased levels of cyclin B1 in TgHD cells suggest shorter G2/M delay, which correlates with abnormal higher proliferation of these cells. 
A

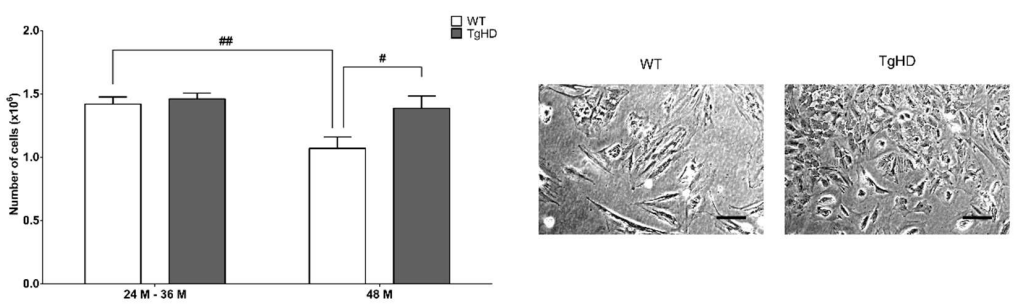

B

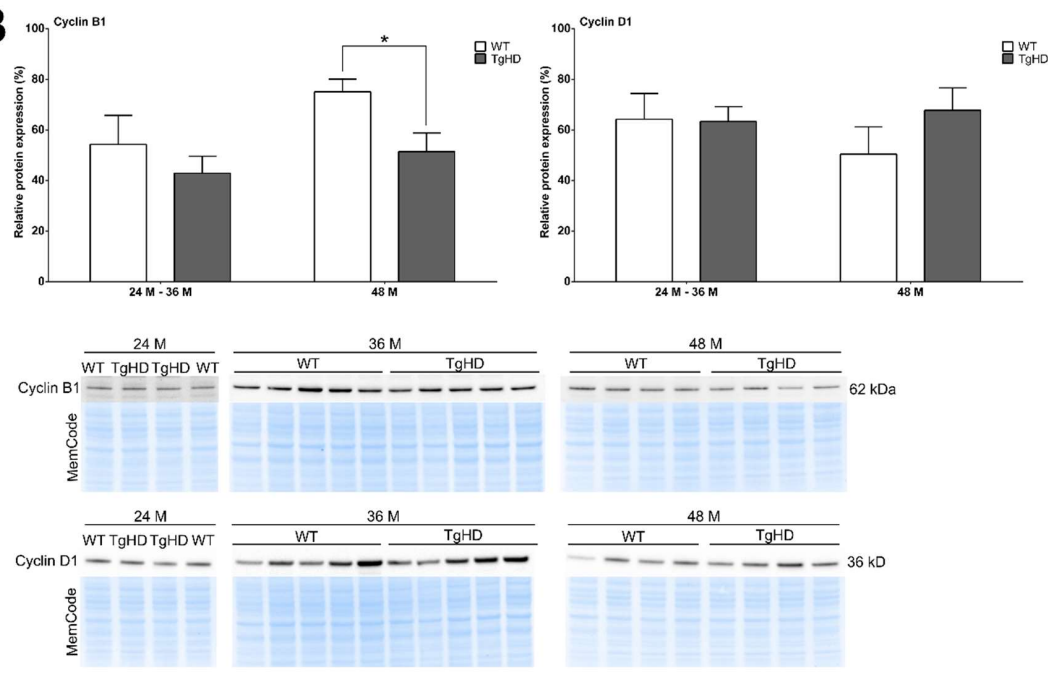

Fig. 2 Altered proliferation of fibroblasts isolated from TgHD animals at the age of $\mathbf{4 8}$ months (A) Equal number (1 mil per $75 \mathrm{~cm}^{2}$ flask) of primary porcine fibroblasts isolated from wild type (WT) and transgenic (TgHD) animals at the pre-onset age (24 M and $36 \mathrm{M}, \mathrm{n}=7 \mathrm{WT}$ and $7 \mathrm{TG})$ and at the age of 48 months ( $48 \mathrm{M}, \mathrm{n}=5 \mathrm{WT}$ and $4 \mathrm{TgHD}$ ) were seeded and cultured in normal culture conditions. The number of viable cells was counted on the $3^{\text {rd }}$ day of cultivation. Values in graph indicate mean of remaining viable cells with SEM. $\# P<0.05, \# \# P<0.01$, two-way ANOVA followed by post hoc Sidak comparison test. An image of representative WT and TgHD cells isolated from 48 months old animals were taken on the 3rd day of cultivation using phase contrast microscope equipped with digital camera. Scale bar is $10 \mu \mathrm{m}$. (B) The expression of cyclin B1 and D1 in primary porcine fibroblasts isolated from WT and TgHD animals at the pre-onset age ( $24 \mathrm{M}$ and $36 \mathrm{M}, \mathrm{n}=7 \mathrm{WT}$ and $7 \mathrm{TgHD})$ and at the age of 48 months $(48 \mathrm{M}, \mathrm{n}=5$ WT and 4 TgHD) analysed by Western blot analysis. Representative Western blots with anti-cyclin B1 antibody and anti-cyclin D1 antibody with total protein loading control obtained by MemCode. Graphs showing cyclin B1 and cyclin D1 expression in the asynchronous population of cells are normalized to total protein obtained by MemCode. Values in graphs indicate percentage of relative protein expression with SEM. $* P<0.05, t$ test. 


\section{Early onset of age-related DNA damage in TgHD fibroblasts}

Increased oxidative stress and altered expression of genes associated with DNA repair and ROSdefence might influence the integrity of cellular DNA. The specific effect of mitochondrial dismutase and general ROS motivated a specific quantification of mitochondrial DNA (mtDNA) damage and nuclear DNA (nDNA) damage. This was performed using an in-house developed qPCR-based method that determines the integrity of template DNA by its ability to be cleaved by a restriction enzyme [29].

The results revealed that nDNA damage level increased significantly with age in WT fibroblasts $(P=0.002, t$ test $)$ (Fig. 3A). Interestingly, there was no age-mediated increase in the level of nDNA damage in TgHD fibroblasts, but this was due to increased DNA damage level already in the presymptomatic age compared to WT ( $P=0.028, t$ test $)$. On the other hand, the integrity of mtDNA did not display any age or genotype difference. There was a tendency to lower mtDNA damage in TgHD in the youngest cohort (Fig. 3B). In summary, the nDNA/mtDNA damage profile in fibroblasts from pre-symptomatic TgHD minipigs is reminiscent of the situation in PBMCs from HD patients [25].
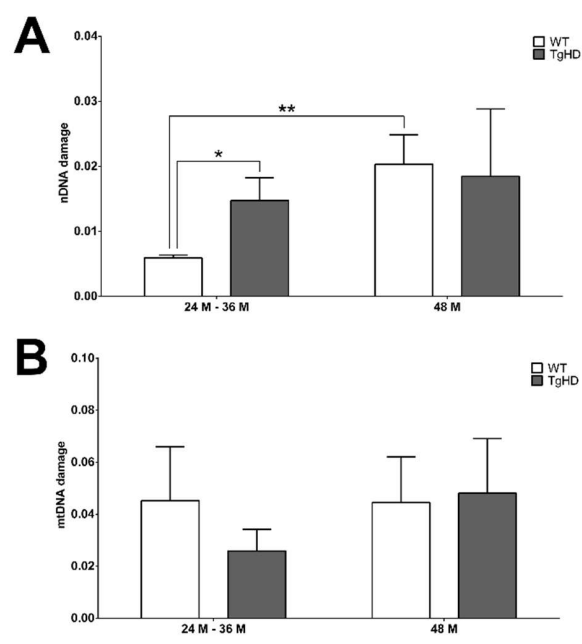

Fig. 3 Increased DNA damage in TgHD fibroblasts at pre-symptomatic age.

The levels of (A) nuclear DNA damage (nDNA damage) and (B) mitochondrial DNA damage (mtDNA damage) in primary porcine fibroblasts isolated from wild type (WT) and transgenic (TgHD) animals at the pre-onset age ( $24 \mathrm{M}-36 \mathrm{M}, \mathrm{n}=7 \mathrm{WT}$ and $7 \mathrm{TgHD}$ ) and at the age of 48 months ( $48 \mathrm{M}, \mathrm{n}=4 \mathrm{WT}$ and $4 \mathrm{TgHD})$. Values in graph indicate mean of damaged DNA with SEM. $* P<0.05, * * P<0.01, t$ test. 


\section{Altered Calcein AM uptake and normal viability in fibroblasts from TgHD minipigs of different age}

Next, we tested Calcein AM uptake by cells to study the permeability of the cell membrane. Lipophilic non-fluorescent Calcein AM freely diffuses through the plasma membrane to the cytosol, where it is hydrolysed by endogenous esterase to fluorescent Calcein which cannot exit the cell, thus its fluorescent intensity can be measured in the cytosol [35]. Our results of fluorescent Calcein intensity spectrophotometry measurements in the cells at the time point of 120 minutes revealed significant difference between TgHD and WT fibroblasts isolated from 48-months-old animals (two-way ANOVA, Genotype $P=0.013, F=7.611$; Sidak post hoc test $P=0.011$ ) (Fig. 4A). Furthermore, Calcein AM uptake is markedly decreased with age in TgHD fibroblasts (two-way ANOVA, Age $P=0.027, F=5.811$; Sidak post hoc test $P=0.007$ ) (Fig. 4A).

To further investigate whether decreased Calcein fluorescence in TgHD fibroblasts isolated from 48-months-old animals could be caused by decreased cell viability, we performed Alamar Blue test of viability (Fig. 4B). Alamar Blue test did not show any differences between TgHD and WT fibroblasts suggesting there are no alterations in viability. These results indicate that TgHD fibroblasts isolated at the age of 48 months have altered permeability but not viability.
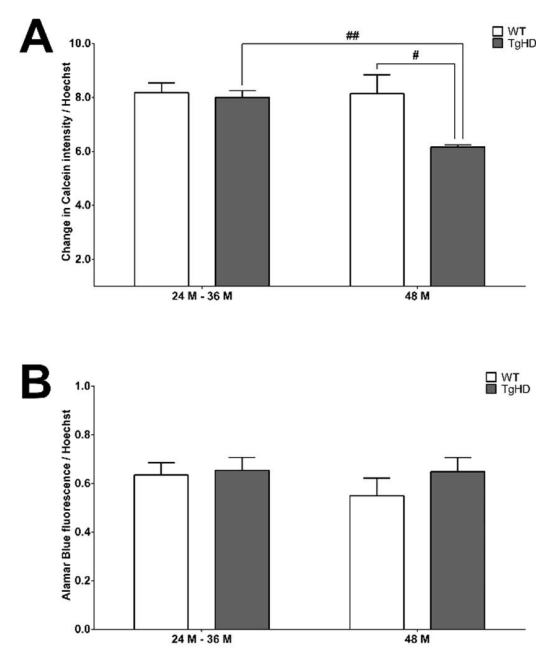

Fig. 4 Calcein AM uptake is altered in TgHD fibroblasts at the age of 48 months

(A) Primary porcine fibroblasts isolated from wild type (WT) and transgenic (TgHD) animals at the pre-onset age ( $24 \mathrm{M}-36 \mathrm{M}, \mathrm{n}=7 \mathrm{WT}$ and $7 \mathrm{TgHD})$ and at the age of 48 months ( $48 \mathrm{M}, \mathrm{n}=4 \mathrm{WT}$ and $4 \mathrm{TgHD})$. Values in graph indicate mean of the change in Calcein fluorescent intensity normalised to Hoechst intensity with SEM. $\# P<0.05, \# \# P<0.01$ two-way ANOVA followed by post hoc Sidak comparison test. (B) Graph showing viability of the cells by the measurement of Alamar Blue fluorescence normalised to Hoechst staining of primary porcine fibroblasts isolated from wild type (WT) and transgenic (TgHD) animals at the pre-onset age ( $24 \mathrm{M}-36 \mathrm{M}, \mathrm{n}=7 \mathrm{WT}$ and $7 \mathrm{TgHD})$ and at the age of 48 months ( $48 \mathrm{M}, \mathrm{n}=4 \mathrm{WT}$ and $4 \mathrm{TgHD})$. Data were obtained by fluorescent spectrophotometry. Each sample was done in triplicates. Values in graph indicate mean with SEM. 


\section{Discussion}

In this study we examined primary porcine fibroblasts isolated from TgHD minipigs, a unique large animal model of HD, at the pre-symptomatic age and at the age of 48 months when first HD-related behavioural and cognitive differences are observed in TgHD animals [12]. HD is the disease of the whole body, since mtHTT is expressed in most of the cell types. Primary fibroblasts are easily collected noninvasively and can be further cultured for a few passages in contrast to neurons, which are, from adult aging animals, hard to be isolated and further cultured for extensive experiments. TgHD fibroblasts stably express mtHTT with age and throughout generations. This makes these cells a suitable model for studying molecular events occurring in HD.

We observed significantly higher levels of lipid peroxidation in pre-symptomatic and symptomatic stage followed by elevated ROS levels in TgHD cells isolated from older 48 months old minipigs. It is well known, that oxidative stress is elevated in $\mathrm{HD}$, even though it is not clear if it is a consequence or rather a cause of the primary degenerative mechanisms [30-33]. Our results suggest that oxidative stress is one of the initial events that occures in HD fibroblasts. It is still a question whether the timing of these oxidative changes is also mirrored in the HD brain (particularly the striatum).

Furthermore, TgHD fibroblasts from 48 months old animals showed upregulation of NEIL3 gene encoding protein capable of repairing DNA damaged by oxidative stress in the base excision repair (BER) pathway. NEIL3 is particularly attributed to the repair of hydantoin-derived lesions from single stranded DNA [36-39], and is specifically expressed in proliferating cells (e.g. stem cells, progenitor cells, other proliferating cells). NEIL3 is expressed in proliferating cells during S phase [40,41]. It is involved in replication associated repair thus it is needed for proper cellular proliferation. It has been shown that NEIL3-deficient cells display decreased proliferation [42]. Furthermore, NEIL3 was found to be overexpressed in various rapidly proliferating cancer cells [43]. Here, increased NEIL3 upregulation is found in TgHD fibroblasts from 48 months minipigs, which correlated with the higher proliferation rate, compared to the situation in WT fibroblasts from minipigs of the same age.

Despite the higher proliferation rate of TgHD fibroblasts representing symptomatic age, this is not attributed to increased genome integrity. Rather, the age-associated increase in nDNA damage, as exemplified in WT porcine fibroblasts appears to be accelerated in TgHD. Illuzzi and colleagues $[44,45]$ reported that expression of mtHTT fragment induces DNA damage response pathway which precede the appearance of detectable HTT aggregates. The situation is reminiscent of PBMCs, were elevated nDNA damage was identified as a novel biomarker of $\mathrm{HD}$ and correlated with Total functional score, the established predictor of disease progression. In comparison, primary fibroblasts from HD patients do not demonstrate increased nDNA damage but in contrast increased mtDNA damage (unpublished observations). These differences are probably ascribed to a combination of differences in organism, tissue, ability of mutation to undergo somatic expansions, and type of mtHTT (N-terminal fragment causing gain of function versus mutated full-length protein causing a combined gain of function/loss of function). We have previously shown higher fragmentation of mtHTT with age in cortex tissues from these TgHD minipigs at the age of 9 to 24 months [11]. It has been also shown, that mtHTT expression in neurons not only causes nuclear DNA damage but further promotes it by impairing components of DNA repair pathway [46]. Another study reported defects of DNA damage signalling and repair in irradiated human HD skin fibroblast [47]. We could not identify altered integrity of nDNA or mtDNA in cortex of TgHD [12]. 
Specific feature of aging is loss of proliferative capacity [48]. In this study we show that primary porcine fibroblasts isolated from TgHD animals show discrepancies in the normal proliferative pattern. Recently, it has been clearly demonstrated that cells expressing mutated huntingtin have significantly increased proliferation rate [49]. In addition, it has been observed that cells expressing mutated huntingtin differ in cell morphology, growth and differentiation [49-51]. These findings along with our results suggest that abnormal higher proliferation is a feature of early HD pathology and it is caused by the action of mutated huntingtin protein.

Our results also show significantly decreased levels of cyclin B1 and slightly (non-significant) increased levels of cyclin D1 in TgHD fibroblasts isolated at the age of 48 months, which further support the abnormal higher proliferation of these cells. Cyclins B1 and D1 are important factors in cell cycle proliferation. Transition from quiescence to proliferative growth (G0/G1 to $\mathrm{S}$ ) is largely regulated by the D-type cyclins whereas cyclin B regulates the transition of G2/M [52]. Cyclin B1 also translocates to mitochondria where it activates the "mitochondrial checkpoint", initiating cell cycle delay, and later the restart of the cell cycle, as a response to oxidative stress, to avoid aberrant proliferation and protect the mitochondrial and nuclear genome integrity [34]. This mitochondrial checkpoint is regulated by manganese superoxide dismutase (SOD2 or MnSOD) $[34,53]$.

SOD2 is a key mitochondrial antioxidant enzyme that catalyses the conversion of reactive superoxide to hydrogen peroxide [54]. SOD2 activity is critical for maintenance of quiescence to prevent aberrant proliferation [53]. Cancer cells display a significant decrease in SOD2 activity, and proliferation of cancer cells can be delayed by SOD2 overexpression [55-57]. 47. Here we found this gene to be upregulated in TgHD cells from 48-months-old animals along with increased levels of oxidative stress, and significantly increased proliferation rates. Because overexpression of SOD2 should suppress accumulation of oxidative stress [34] the activity of SOD2 might be compromised in TgHD fibroblasts from 48-months-old animals. We performed SOD2 activity assay and found, as we assumed, SOD2 activity to be lower even though not significantly (Supplementary Fig. S2). For better understanding of the mechanisms causing abnormal proliferation and oxidative stress in cells expressing mutated huntingtin more experiments are needed.

In this study we further show that the Calcein fluorescence is significantly lower in TgHD cells isolated from symptomatic animals (at the age of 48 months), whereas the cell viability of all examined groups remains intact. Non-fluorescent Calcein AM can freely diffuse across the plasma membrane into the cell where it is hydrolysed by esterases into highly fluorescent Calcein that stays in the cellular interior thus, cellular permeability can be measured according to its fluorescence. This indicates that TgHD cells isolated from 48-months-old animals have altered plasmatic membrane permeability. Calcein AM efflux has been used for several studies focusing on functional detection of multidrug resistance in live cells in the presence of multidrug efflux transporters $[35,58,59]$. It has been shown that the fluorescence of Calcein is decreased in cells expressing transporters compared to cells which do not express them [35]. Thus, Calcein fluorescence may be an indicator of ATP transporter activity. Huntingtin protein is largely distributed in the cytoplasm and directly interacts with phospholipid membranes [60-62]. Interestingly, Suopanki and colleagues [63] have shown that soluble WT and even mutated oligomeric huntingtin proteins cause increased membrane permeability, whereas fibrillar structures of mtHTT protein decrease membrane permeability. It has been suggested that dysfunction of membrane trafficking mediated by mtHTT could be one of the main cause of neurotoxicity in HD [64]. Further studies regarding 
the altered cellular permeability in cells expressing mtHTT are necessary in order to determine its role in HD neurotoxicity.

In summary, our results suggest that oxidative stress in primary fibroblasts isolated from symptomatic TgHD minipigs is accompanied by other molecular events such as altered proliferation and cellular permeability as well as earlier nuclear DNA damage accumulation in these cells.

The results of this study show that DNA damage and oxidative stress coincides with abnormal proliferation and altered permeability of primary fibroblasts from the TgHD minipig model of HD. This study and earlier evidence support the premise that primary fibroblasts expressing mtHTT correlate in some extend with striatal cell lines [49] and that TgHD minipigs serve as a promising large animal model of HD. The age of 48 months manifests as a certain age of onset in developing molecular phenotype of HD in this large animal model. Furthermore, our results suggest that primary cells isolated from this model offer an opportunity to study and better understand the molecular mechanisms occurring gradually in HD pathophysiology with age. Biomarkers found in these cells could serve for evaluation of efficiency when whole-body HD treatment will be applied. 


\section{List of abbreviations}

HD: Huntingtin's disease

TgHD: transgenic for the N-terminal part of human mutated huntingtin

WT: wild type control

HTT: huntingtin

mtHTT: mutated huntingtin

enHTT: endogenous huntingtin

ROS: reactive oxygen species

mtDNA: mitochondrial DNA

nDNA: nuclear DNA

BER: base excision repair

NEIL3: nei like DNA glycosylase 3

SOD2: superoxide dismutase 2

PBMCs: Peripheral blood mononuclear cells

\section{Declarations:}

\section{Ethics approval and consent to participate}

Approval from the Committee of Institute of Animal Physiology and Genetics and the State Veterinary Administration of the Czech Republic was obtained prior to all animal-related studies (protocol \# 53/2015).

\section{Consent for publication}

Not applicable.

\section{Availability of data and materials}

The datasets used and/or analysed during the current study are available from the corresponding author on reasonable request.

\section{Competing interests}

The authors declare that they have no competing interests

\section{Funding}

This work was supported by PIGMOD Center's sustainability program: National Sustainability Programme, project number LO1609 (Czech Ministry of Education, Youth and Sports), CHDI foundation, project number A-5378, and Czech-Norwegian research programme, project number 7F14308.

\section{Authors' contributions}

PS, LE, ZE designed the research; PS, GA, MV, JK performed the experiments; PS analysed the data, JM supervised the research, PS, LE, and ZE wrote the manuscript. All authors read and approved the final manuscript.

\section{Acknowledgements}

Not applicable. 


\section{References}

1. Stack EC, Ferrante RJ. Huntington's disease: progress and potential in the field. Expert Opin Investig Drugs. 2007;16(12):1933-53. doi: 10.1517/13543784.16.12.1933.

2. Bates G. Huntingtin aggregation and toxicity in Huntington's disease. Lancet. 2003;361(9369):1642-4. doi: 10.1016/S0140-6736(03)13304-1.

3. Cooper J. Truncated N-terminal fragments of huntingtin with expanded glutamine repeats form nuclear and cytoplasmic aggregates in cell culture. Hum Mol Genet. 1998;7(5):78390. doi: $10.1093 / \mathrm{hmg} / 7.5 .783$.

4. Hackam AS, Singaraja R, Wellington CL, Metzler M, McCutcheon K, Zhang T, et al. The Influence of Huntingtin Protein Size on Nuclear Localization and Cellular Toxicity. J Cell Biol. 1998;141(5):1097-105. doi: 10.1083/jcb.141.5.1097.

5. Martindale D, Hackam A, Wieczorek A, Ellerby L, Wellington C, McCutcheon K, et al. Length of huntingtin and its polyglutamine tract influences localization and frequency of intracellular aggregates. Nat Genet. 1998;18(2):150-4. doi: 10.1038/ng0298-150.

6. Baxa M, Hruska-Plochan M, Juhas S, Vodicka P, Pavlok A, Juhasova J, et al. A Transgenic Minipig Model of Huntington's Disease. J Huntingtons Dis. 2013;2(1):47-68. doi: 10.3233/JHD-130001.

7. Macakova M, Bohuslavova B, Vochozkova P, Pavlok A, Sedlackova M, Vidinska D, et al Mutated Huntingtin Causes Testicular Pathology in Transgenic Minipig Boars. Neurodegener Dis. 2016;16(3-4):245-59. doi: 10.1159/000443665.

8. Jozefovicova M, Herynek V, Jiru F, Dezortova M, Juhasova J, Juhas S, et al. 31P MR Spectroscopy of the Testes and Immunohistochemical Analysis of Sperm of Transgenic Boars Carried N-terminal Part of Human Mutated Huntingtin. Česká a Slov Neurol a Neurochir. 2015;78/111(S2):28-33. doi: 10.14735/amcsnn20152S28.

9. Krizova J, Stufkova H, Rodinova M, Macakova M, Bohuslavova B, Vidinska D, et al. Mitochondrial Metabolism in a Large-Animal Model of Huntington Disease: The Hunt for Biomarkers in the Spermatozoa of Presymptomatic Minipigs. Neurodegener Dis. 2017;17(4-5):213-26. doi: 10.1159/000475467.

10. Valekova I, Jarkovska K, Kotrcova E, Bucci J, Ellederova Z, Juhas S, et al. Revelation of the IFN $\alpha$, IL-10, IL- 8 and IL- $1 \beta$ as promising biomarkers reflecting immuno-pathological mechanisms in porcine Huntington's disease model. J Neuroimmunol. 2016;293:71-81. doi: 10.1016/j.jneuroim.2016.02.012.

11. Vidinska D, Vochozkova P, Smatlikova P, Ardan T, Klima J, Juhas S, et al. Gradual Phenotype Development in Huntington Disease Transgenic Minipig Model at 24 Months of Age. Neurodegener Dis. 2018;18(2-3):107-19. doi: 10.1159/000488592.

12. Askeland G, Rodinova M, Stufkova H, Dosoudilova Z, Baxa M, Smatlikova P, et al. A transgenic minipig model of Huntington's disease shows early signs of behavioral and molecular pathologies. Dis Model Mech. 2018;11(10):dmm.035949. doi: $10.1242 / \mathrm{dmm} .035949$.

13. Bhide PG, Day M, Sapp E, Schwarz C, Sheth A, Kim J, et al. Expression of normal and mutant huntingtin in the developing brain. J Neurosci. 1996;16(17):5523-35.

14. Tasset I, Sanchez F, Tunez I. The molecular bases of Huntington's disease: the role played by oxidative stress. Rev Neurol. 2009;49(8):424-9.

15. Stack EC, Matson WR, Ferrante RJ. Evidence of Oxidant Damage in Huntington's Disease: Translational Strategies Using Antioxidants. Ann N Y Acad Sci. 
2008;1147(1):79-92. doi: 10.1196/annals.1427.008.

16. Kumar A, Ratan RR. Oxidative Stress and Huntington's Disease: The Good, the Bad, and the Ugly. J Huntingtons Dis. 2016;5(3):217-37. doi: 10.3233/JHD-160205.

17. Burdon RH, Gill V. Cellularly Generated Active Oxygen Species and Hela Cell Proliferation. Free Radic Res Commun. 1993;19(3):203-13. doi: 10.3109/10715769309111603.

18. Sundaresan M, Yu Z-X, Ferrans VJ, Irani K, Finkel T. Requirement for Generation of $\mathrm{H}(2) \mathrm{O}(2)$ for Platelet-Derived Growth Factor Signal Tran sduction. Science (80- ). 1995;270(5234):296-9. doi: 10.1126/science.270.5234.296.

19. Browne SE, Beal MF. The Energetics of Huntington's Disease. Neurochem Res. 2004;29(3):531-46. doi: 10.1023/B:NERE.0000014824.04728.dd.

20. Browne SE. Mitochondria and Huntington's Disease Pathogenesis. Ann N Y Acad Sci. 2008;1147(1):358-82. doi: 10.1196/annals.1427.018.

21. Reddy PH, Mao P, Manczak M. Mitochondrial structural and functional dynamics in Huntington's disease. Brain Res Rev. 2009;61(1):33-48. doi: 10.1016/j.brainresrev.2009.04.001.

22. Damiano M, Galvan L, Déglon N, Brouillet E. Mitochondria in Huntington's disease. Biochim Biophys Acta - Mol Basis Dis. 2010;1802(1):52-61. doi: 10.1016/j.bbadis.2009.07.012.

23. Kim J, Moody JP, Edgerly CK, Bordiuk OL, Cormier K, Smith K, et al. Mitochondrial loss, dysfunction and altered dynamics in Huntington's disease. Hum Mol Genet. 2010;19(20):3919-35. doi: 10.1093/hmg/ddq306.

24. Hands S, Sajjad MU, Newton MJ, Wyttenbach A. In vitro and in vivo aggregation of a fragment of huntingtin protein directly causes free radical production. J Biol Chem. 2011;286(52):44512-20. doi: 10.1074/jbc.M111.307587.

25. Askeland G, Dosoudilova Z, Rodinova M, Klempir J, Liskova I, Kusnierczyk A, et al. Increased nuclear DNA damage precedes mitochondrial dysfunction in peripheral blood mononuclear cells from Huntington's disease patients. Sci Rep. 2018;8(1):9817. doi: 10.1038/s41598-018-27985-y.

26. Moshell AN, Barrett SF, Tarone RE, Robbins JH. Radiosensitivity in Huntington's disease: implications for pathogenesis and presymptomatic diagnosis. Lancet. 1980;315(8158):9-11. doi: 10.1016/S0140-6736(80)90550-4.

27. Robison SH, Bradley WG. DNA damage and chronic neuronal degenerations. J Neurol Sci. 1984; doi: 10.1016/0022-510X(84)90051-0.

28. Scudiero DA, Meyer SA, Clatterbuck BE, Tarone RE, Robbins JH. Hypersensitivity to Nmethyl-N'-nitro-N-nitrosoguanidine in fibroblasts from patients with Huntington disease, familial dysautonomia, and other primary neuronal degenerations. Proc Natl Acad Sci. 1981;78(10):6451-5. doi: 10.1073/pnas.78.10.6451.

29. Wang W, Scheffler K, Esbensen Y, Eide L. Quantification of DNA Damage by Real-Time qPCR. Methods Mol Biol. 2016;1351:27-32. doi: 10.1007/978-1-4939-3040-1_3.

30. Perluigi M, Poon HF, Maragos W, Pierce WM, Klein JB, Calabrese V, et al. Proteomic analysis of protein expression and oxidative modification in $\mathrm{r} 6 / 2$ transgenic mice: a model of Huntington disease. Mol Cell Proteomics. 2005;4(12):1849-61. doi: 10.1074/mcp.M500090-MCP200.

31. Sorolla MA, Reverter-Branchat G, Tamarit J, Ferrer I, Ros J, Cabiscol E. Proteomic and 
oxidative stress analysis in human brain samples of Huntington disease. Free Radic Biol Med. 2008;45(5):667-78. doi: 10.1016/j.freeradbiomed.2008.05.014.

32. Polidori MC, Mecocci P, Browne SE, Senin U, Beal MF. Oxidative damage to mitochondrial DNA in Huntington's disease parietal cortex. Neurosci Lett. 1999;272(1):53-6. doi: 10.1016/S0304-3940(99)00578-9.

33. Browne SE, Ferrante RJ, Beal MF. Oxidative stress in Huntington's disease. Brain Pathol. 1999;9(1):147-63.

34. Kalen A, Ahmad I, Abdalla M, O’Malley Y, Goswami P, Sarsour E. MnSOD and Cyclin B1 Coordinate a Mito-Checkpoint during Cell Cycle Response to Oxidative Stress. Antioxidants. 2017;6(4):92. doi: 10.3390/antiox6040092.

35. Sharom FJ, Siarheyeva A. Functional assays for identification of compounds that interact with P-gp. In: Colabufo NA, editor. Vol. 37/661, Multidrug Resistance: Biological and Pharmaceutical Advances in Antitumour Treatment. Research Signpost; 2008. p. 261-290.

36. Neurauter CG, Luna L, Bjørås M. Release from quiescence stimulates the expression of human NEIL3 under the control of the Ras dependent ERK-MAP kinase pathway. DNA Repair (Amst). 2012;11(4):401-9. doi: 10.1016/j.dnarep.2012.01.007.

37. Takao M, Oohata Y, Kitadokoro K, Kobayashi K, Iwai S, Yasui A, et al. Human Nei-like protein NEIL3 has AP lyase activity specific for single-stranded DNA and confers oxidative stress resistance in Escherichia coli mutant. Genes to Cells. 2009;14(2):261-70. doi: 10.1111/j.1365-2443.2008.01271.x.

38. Hildrestrand GA, Neurauter CG, Diep DB, Castellanos CG, Krauss S, Bjoras M, et al. Expression patterns of Neil3 during embryonic brain development and neoplasia. BMC Neurosci. 2009;10(1):45. doi: 10.1186/1471-2202-10-45.

39. Rolseth V, Runden-Pran E, Luna L, McMurray C, Bjørås M, Ottersen OP. Widespread distribution of DNA glycosylases removing oxidative DNA lesions in human and rodent brains. DNA Repair (Amst). 2008;7(9):1578-88. doi: 10.1016/j.dnarep.2008.06.007.

40. Liu M, Bandaru V, Holmes A, Averill AM, Cannan W, Wallace SS. Expression and purification of active mouse and human NEIL3 proteins. Protein Expr Purif. 2012;84(1):130-9. doi: 10.1016/j.pep.2012.04.022.

41. Liu M, Bandaru V, Bond JP, Jaruga P, Zhao X, Christov PP, et al. The mouse ortholog of NEIL3 is a functional DNA glycosylase in vitro and in vivo. Proc Natl Acad Sci. 2010;107(11):4925-30. doi: 10.1073/pnas.0908307107.

42. Reis A, Hermanson O. The DNA glycosylases OGG1 and NEIL3 influence differentiation potential, proliferation, and senescence-associated signs in neural stem cells. Biochem Biophys Res Commun. 2012;423(4):621-6. doi: 10.1016/j.bbrc.2012.04.125.

43. Kauffmann A, Rosselli F, Lazar V, Winnepenninckx V, Mansuet-Lupo A, Dessen P, et al. High expression of DNA repair pathways is associated with metastasis in melanoma patients. Oncogene. 2008;27(5):565-73. doi: 10.1038/sj.onc.1210700.

44. Illuzzi J, Yerkes S, Parekh-Olmedo H, Kmiec EB. DNA breakage and induction of DNA damage response proteins precede the appearance of visible mutant huntingtin aggregates. J Neurosci Res. 2009;87(3):733-47. doi: 10.1002/jnr.21881.

45. Illuzzi JL, Vickers C a., Kmiec EB. Modifications of p53 and the DNA damage response in cells expressing mutant form of the protein huntingtin. J Mol Neurosci. 2011;45(2):256-68. doi: 10.1007/s12031-011-9516-4

46. Enokido Y, Tamura T, Ito H, Arumughan A, Komuro A, Shiwaku H, et al. Mutant 
huntingtin impairs Ku70-mediated DNA repair. J Cell Biol. 2010;189(3):425-43. doi: 10.1083/jcb.200905138.

47. Ferlazzo ML, Sonzogni L, Granzotto A, Bodgi L, Lartin O, Devic C, et al. Mutations of the Huntington's disease protein impact on the ATM-dependent signaling and repair pathways of the radiation-induced DNA double-strand breaks: Corrective effect of statins and bisphosphonates. Mol Neurobiol. 2014;49(3):1200-11. doi: 10.1007/s12035-0138591-7.

48. Harris N, Costa V, MacLean M, Mollapour M, Moradas-Ferreira P, Piper PW. Mnsod overexpression extends the yeast chronological (G0) life span but acts independently of Sir $2 p$ histone deacetylase to shorten the replicative life span of dividing cells. Free Radic Biol Med. 2003;34(12):1599-606. doi: 10.1016/S0891-5849(03)00210-7.

49. Singer E, Walter C, Weber JJ, Krahl A-C, Mau-Holzmann UA, Rischert N, et al. Reduced cell size, chromosomal aberration and altered proliferation rates are characteristics and confounding factors in the STHdh cell model of Huntington disease. Sci Rep. 2017;7(1):16880. doi: 10.1038/s41598-017-17275-4.

50. Reis SA, Thompson MN, Lee J-M, Fossale E, Kim H-H, Liao JK, et al. Striatal neurons expressing full-length mutant huntingtin exhibit decreased $\mathrm{N}$-cadherin and altered neuritogenesis. Hum Mol Genet. 2011;20(12):2344-55. doi: 10.1093/hmg/ddr127.

51. Lim D, Fedrizzi L, Tartari M, Zuccato C, Cattaneo E, Brini M, et al. Calcium Homeostasis and Mitochondrial Dysfunction in Striatal Neurons of Huntington Disease. J Biol Chem. 2008;283(9):5780-9. doi: 10.1074/jbc.M704704200.

52. Bertoli C, Skotheim JM, de Bruin RAM. Control of cell cycle transcription during G1 and S phases. Nat Rev Mol Cell Biol. 2013;14(8):518-28. doi: 10.1038/nrm3629.

53. Sarsour EH, Venkataraman S, Kalen AL, Oberley LW, Goswami PC. Manganese superoxide dismutase activity regulates transitions between quiescent and proliferative growth. Aging Cell. 2008;7(3):405-17. doi: 10.1111/j.1474-9726.2008.00384.x.

54. Weisiger RA, Fridovich I. Proviruses: Site of Synthesis. Nature. 1973;241(5386):166-166. doi: $10.1038 / 241166 \mathrm{a} 0$.

55. Li N, Oberley TD, Oberley LW, Zhong W. Inhibition of cell growth in NIH/3T3 fibroblasts by overexpression of manganese superoxide dismutase: Mechanistic studies. J Cell Physiol. 1998;175(3):359-69. doi: 10.1002/(SICI)10974652(199806)175:3<359::AID-JCP14>3.0.CO;2-0.

56. Ough M, Lewis A, Zhang Y, Hinkhouse MM, Ritchie JM, Oberley LW, et al. Inhibition of Cell Growth by Overexpression of Manganese Superoxide Dismutase (MnSOD) in Human Pancreatic Carcinoma. Free Radic Res. 2004;38(11):1223-33. doi: $10.1080 / 10715760400017376$.

57. Venkataraman S, Jiang X, Weydert C, Zhang Y, Zhang HJ, Goswami PC, et al. Manganese superoxide dismutase overexpression inhibits the growth of androgenindependent prostate cancer cells. Oncogene. 2005;24(1):77-89. doi: 10.1038/sj.onc. 1208145 .

58. Lebedeva I V., Pande P, Patton WF. Sensitive and Specific Fluorescent Probes for Functional Analysis of the Three Major Types of Mammalian ABC Transporters. PLoS One. 2011;6(7):e22429. doi: 10.1371/journal.pone.0022429.

59. Litman T, Brangi M, Hudson E, Fetsch P, Abati A, Ross DD, et al. The multidrug-resistant phenotype associated with overexpression of the new ABC half-transporter, MXR (ABCG2). J Cell Sci. 2000;113:2011-21. doi: 10.1055/s-0028-1096972. 
60. DiFiglia M, Sapp E, Chase K, Schwarz C, Meloni A, Young C, et al. Huntingtin is a cytoplasmic protein associated with vesicles in human and rat brain neurons. Neuron. 1995;14(5):1075-81. doi: 10.1016/0896-6273(95)90346-1.

61. Velier J, Kim M, Schwarz C, Kim TW, Sapp E, Chase K, et al. Wild-Type and Mutant Huntingtins Function in Vesicle Trafficking in the Secretory and Endocytic Pathways. Exp Neurol. 1998;152(1):34-40. doi: 10.1006/exnr.1998.6832.

62. Kegel KB, Kim M, Sapp E, McIntyre C, Castaño JG, Aronin N, et al. Huntingtin expression stimulates endosomal-lysosomal activity, endosome tubulation, and autophagy. J Neurosci. 2000;20(19):7268-78. doi: 10.1523/jneurosci.0800-08.2008.

63. Suopanki J, Götz C, Lutsch G, Schiller J, Harjes P, Herrmann A, et al. Interaction of huntingtin fragments with brain membranes - Clues to early dysfunction in Huntington's disease. J Neurochem. 2006;96(3):870-84. doi: 10.1111/j.1471-4159.2005.03620.x.

64. Li S-H, Li X-J. Huntington and its Role in Neuronal Degeneration. Neurosci. 2004;10(5):467-75. doi: 10.1177/1073858404266777. 


\section{Supplementary figures}

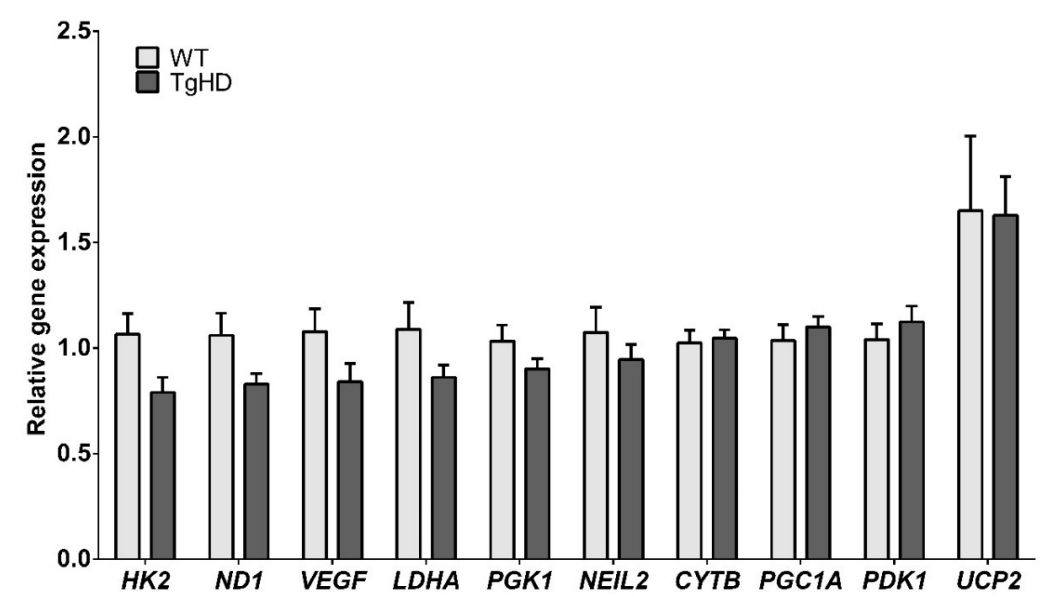

Fig. S1 Relative gene expression in transgenic and wild type primary porcine fibroblasts isolated from 48 months old animals.

Graph show relative expression of UCP2, HK2, ND1, VEGF, LDHA, PGK1, NEIL2, CYTB, PGC1A, PDK1 genes in primary fibroblasts isolated from transgenic $(\mathrm{TgHD}, \mathrm{n}=4)$ and wild type $(\mathrm{WT}, \mathrm{n}=4)$ minipigs at the age of 48 months. Data were obtained by qPCR analysis and normalized to B2M and ACTB porcine housekeeping genes. Data represent the average from 5 independent experiments. Values in graph indicate mean with SEM.

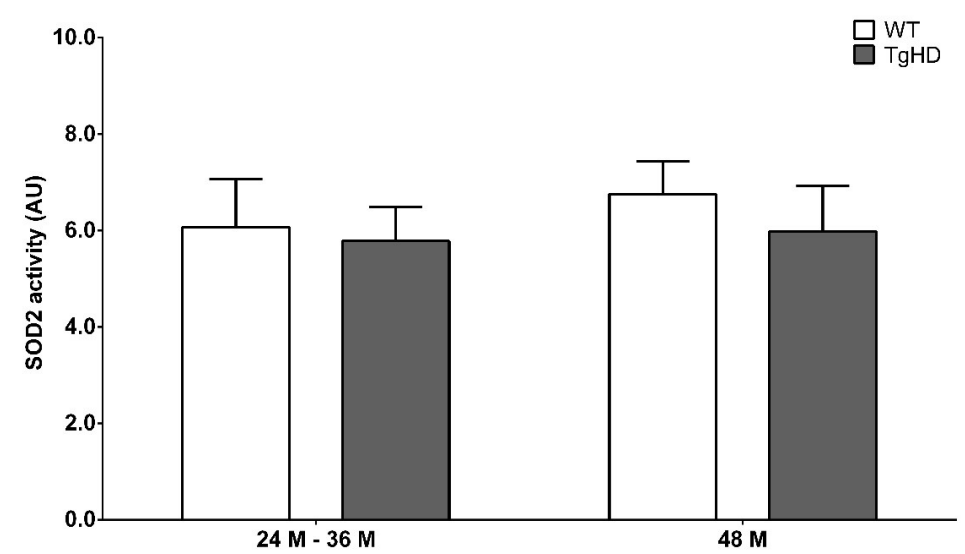

Fig. S2 SOD2 activity in WT and TgHD porcine fibroblasts

SOD2 activity measured by SOD determination kit (Sigma, \#19160) in mitochondrial fraction of primary porcine fibroblasts isolated from wild type (WT) and transgenic (TgHD) animals at the pre-onset age (24$36 \mathrm{M}, \mathrm{n}=7 \mathrm{WT}$ and 7 TgHD) and at the age of 48 months (48 M, n=4 WT and 4 TgHD). Values in graph indicate mean with SEM. 